\title{
The importance of ground magnetic data in specifying the state of magnetosphere- ionosphere coupling: a personal view
}

\author{
Y. Kamide ${ }^{1,2^{*}}$ and Nanan Balan ${ }^{3}$
}

\begin{abstract}
In the history of geomagnetism, geoelectricity and space science including solar terrestrial physics, ground magnetic records have been demonstrated to be a powerful tool for monitoring the levels of overall geomagnetic activity. For example, the Kp and ap indices having perhaps the long-history geomagnetic indices have and are being used as space weather parameters, where " $p$ " stands for "planetary" implying that these indices express average geomagnetic disturbances on the entire Earth in a planetary scale. To quantify the intensity level of geomagnetic storms, however, it is common to rely on the Dst index, which is supposed to show the magnitude of the storm-time ring current. Efforts were also made to inter-calibrate various activity indices. Different indices were proposed to express different aspects of a phenomenon in the near-Earth space. In the early 1980s, several research groups in Japan, Russia, Europe and the US developed the so-called magnetogram-inversion techniques, which were proposed all independently. Subsequent improvements of the magnetogram-inversion algorithms allowed their technology to be applied to a number of different datasets for magnetospheric convection and substorms. In the present review, we demonstrate how important it was to make full use of ground magnetic data covering a large extent in both latitudinal and longitudinal directions. It is now possible to map a number of electrodynamic parameters in the polar ionosphere on an instantaneous basis. By applying these new inverse methods to a number of ground-based geomagnetic observations, it was found that two basic elements in spatial patterns can be viewed as two physical processes for solar wind-magnetosphere energy coupling.
\end{abstract}

Keywords: Geomagnetic field, Magnetic storm and substorm, Magnetosphere-ionosphere coupling, Geomagnetic activity indices, Auroral electrojets, Magnetogram inversion, Solar wind, Loading-unloading process, Directly driven process

\section{Introduction}

It is the primary purpose of this paper to review concisely how much the use of ground magnetic observations has contributed to our specification of the energy state of the entire Sun-Earth system. The energy the magnetosphere is gaining from the solar wind for magnetospheric substorms and storms can be estimated from the distribution of electric current vectors in space, which are obtained from geomagnetic disturbances observed on the ground. Different indices were proposed to express

\footnotetext{
*Correspondence: kamide@gray.plala.or.jp

2 Rikubetsu Space and Earth Science Museum, Rikubetsu 049-4301, Japan Full list of author information is available at the end of the article
}

different aspects of a particular phenomenon (Sugiura 1964; Mayaud 1980). However, there is no simple way to convert one or one family of geomagnetic indices to other types of different families of indices.

After many years of examining the measured current systems on the basis of the equivalent current systems, several numerical techniques were developed at several institutes independently in recent years, but this concise review deals mainly with the Kamide-Richmond-Matsushita (KRM) and assimilative mapping of ionospheric electrodynamics (AMIE) techniques (Kamide et al. 1981; Richmond and Kamide 1988). Note that it is now possible to map a number of electrodynamic quantities in the entire polar ionosphere on an instantaneous basis. Since 
field-aligned currents are also derivable from the numerical technique, this "remote sensing" method is quite useful in discussing magnetosphere-ionosphere coupling as well. We will briefly discuss implications of the essential concept and practical procedure of the so-called inversion technique, and will list the advantages and the limitations.

\section{Role of ground-based observations}

Although it is in principle impossible to separate accurately the effects of different current sources only through magnetic measurements made on the ground, several powerful inversion techniques were proposed to attempt to separate the effects of different currents in ground magnetic observations. It is also important to clarify the global and local patterns of the ground magnetic perturbations when they are compared with other more direct observations of plasma population, auroras, and electric fields and currents in the ionosphere and magnetosphere.

The logic governing the entire magnetosphere-ionosphere coupling system is shown schematically in Fig. 1, which simplifies real processes that occur as a result of close interactions of electric fields and currents in the system (Kamide and Richmond 1991). In fact, nearly every magnetospheric process bears on the ionosphere in some way, and every ionospheric process depends on the magnetosphere. It is for this reason that a number of geomagneticians attempted to separate the effects of ionospheric currents from those of magnetospheric currents, including field-aligned currents and the ring current.

Until recently, studies were measuring one variable of the entire $\mathrm{M}-\mathrm{I}$ chain, assuming a second variable, and then calculating the third variable. For example, the electric field was measured, the ionospheric conductivity was assumed, and the current was able to be calculated. Such an approach does not necessarily treat the interactive nature of the mechanism and thus the conclusions from such studies are not unique. By repeating this type of practice, one cannot properly address the basic, complicated, nonlinear problems that treat the luminosity of auroras.

New numerical methods (Mishin 1990; Feldstein and Levitin 1986; Glassmeier 1987) were developed, which coincided with the availability of new systematic magnetometer data. Three numerical schemes were designed to compute not only the global distribution of both ionospheric and field-aligned currents, but also the ionospheric electric fields and Joule heating rate (Fayermark 1977; Kisabeth 1979; Baumjohann et al. 1981; Mishin 1990). Once we understand properly the origins of ground magnetic perturbations in terms of various source currents, ground-based observations have an advantage over "more direct" measurements by radars and satellites, since temporal changes in the geomagnetic field are being monitored continuously at a relatively

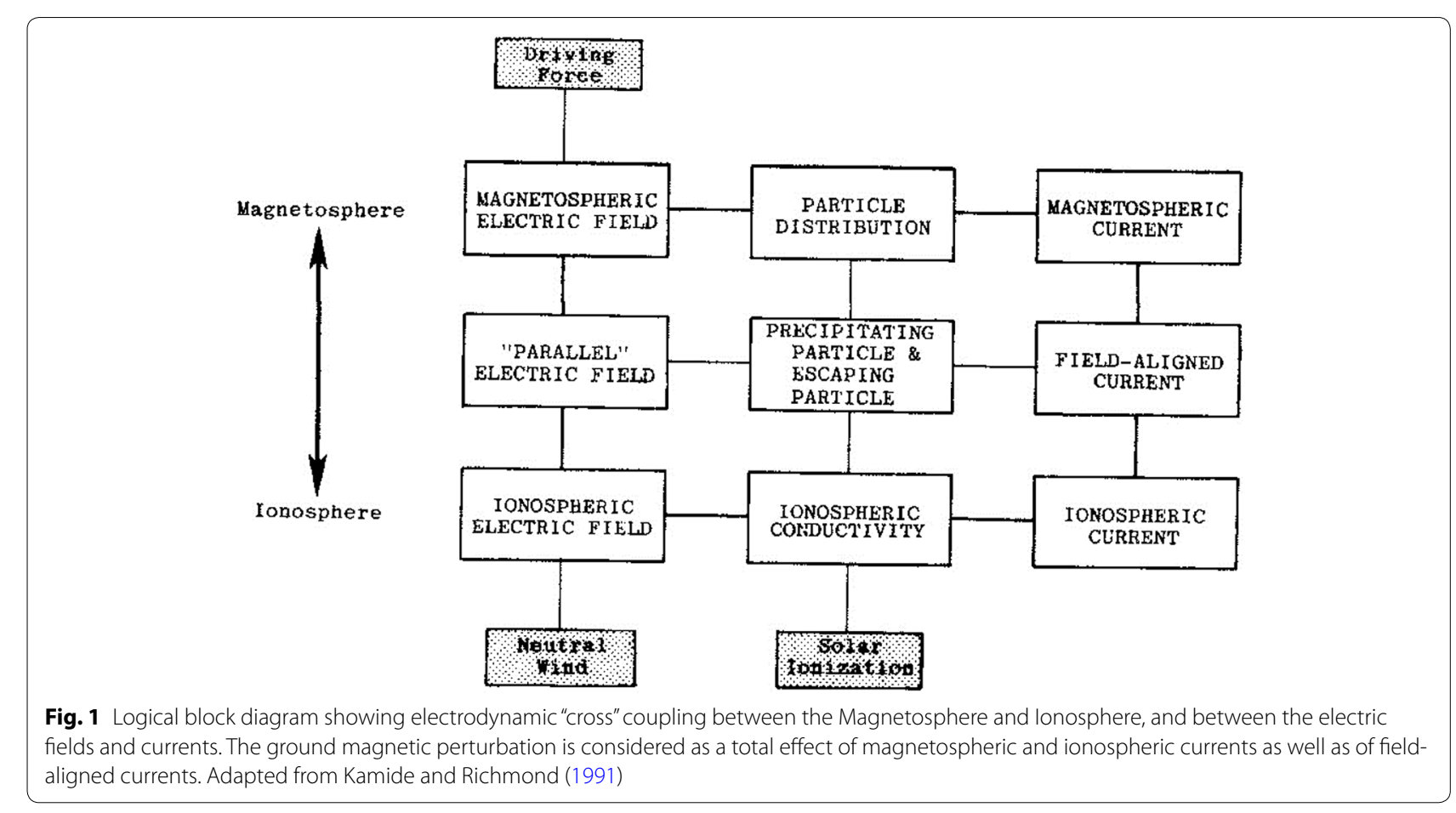


large number of fixed points on the Earth's surface. This contrasts to the intrinsic ambiguity in determining the global three-dimensional current system on an individual basis from single satellite passes, which have difficulties separating temporal and spatial changes. For details on the essence of the magnetogram-inversion techniques, see (Kamide and Baumjohann 1993).

What is outstanding about the new methods, such as KRM and AMIE? The magnetogram inversion is instrumental in unifying the information obtained from synoptic observations of geomagnetic fields with the comprehensive in situ observations by spacecraft in magnetospheric research. Usefulness of this method for unveiling polar phenomena in the ionosphere and the corresponding magnetospheric processes has been verified by numerous studies for individual cases that have used these inversion methods. Some of the successful examples which we report can be found in Kamide (1988).

\section{Applications of magnetogram-inversion techniques to polar substorms}

The magnetogram-inversion techniques have been shown to be quite mighty and powerful in finding even what causes the auroral electrojets, which is one of the most important elements of magnetospheric substorms. In addition, it would become more powerful if it is combined with other innovative techniques and/or other types of observations.

Regarding the auroral electrojets, they are a specific class of concentrated current flows which are most intense in the region of brightest auroral luminosity. This leads us to the simple view that precipitating energetic particles would enhance ionospheric conductivities, and cause auroras, along with carrying field-aligned currents. If this chain of processes were all genuine, then the growth and decay of the auroral electrojets would be determined by those only of the auroras (Kamide and Richmond 1991; Kamide and Kokubun 1996).

Examination of the recently available simultaneous data from coherent and incoherent radars at high latitudes and polar-orbiting satellites, combined with ground magnetic records, has shown that this is not the case at all. Nature told us that the reality is more complicated. There are systematic overlappings in regions in the polar ionosphere where each of the parameters dominates. On the basis of our knowledge of the spatial-temporal relationship of ionospheric electric fields, currents and conductivities with respect to various forms of auroral luminosities at different substorm phases, an attempt was made to show that within the so-called auroral electrojet there are basically two separate regions, an electric-fielddominant part and a conductivity-dominant part. These two components can be viewed as signaling that two dominant physical processes are operative for solar windmagnetosphere energy coupling, i.e., the directly driven and the loading-unloading mechanisms. The relative importance of these two components varies significantly, depending on the substorm phases (Kamide 1988). In the following two subsections, we discuss the role of two basic processes in two different topics.

\section{Electric field and conductivity within the auroral electrojets} In this section, it is important to realize that the region of the intense auroral electrojets is neither completely the same as the region of the highest conductivity nor coincides with that of the largest electric field at all local times. One can find many cases where no bright auroras are present even though the electrojet current there is very intense (Nishida and Kamide 1983). It is also important to note that disturbed intervals, that is, increases in the auroral electrojets, do not automatically mean substorm times, although many papers have relied on an operational definition that if the magnitude of the AE index exceeds some threshold value, say several hundred nanoteslas, that interval must be a substorm interval.

Kamide and Kokubun (1996) showed that there are often spatial shifts among the regions of high conductivity, large electric fields, and intense electrojet currents. In particular, there are an electric-field-dominant part and a conductivity-rich part within the auroral electrojets. The different parts tend to respond in different ways to substorms. These points are described in detail in the following studies:

(a) Radar and particle precipitation (Nishida and Kamide 1983).

(b) Conductivity from dynamics Explorer auroral images (Kamide et al. 1989).

(c) Satellite X-ray images (Ahn et al. 1989).

(d) Combining satellite and radar data with groundbased magnetometer data (Ahn et al. 1992).

\section{Average patterns of ionospheric parameters for different substorm phases}

Among a variety of accomplishments of our inversion technique (KRM assimilated), we will show herewith the average patterns of the electrostatic potential and current vectors for different phases. See Kamide and Kokubun (1996) for the outputs in terms of Joule heating and associated field-aligned currents and for the selection of substorms and KRM magnetogram-inversion technique applied to individual substorms. The average pattern of substorm quantities in the polar ionosphere for the different epochs was determined. 
Figures 2 and 3 show the global distribution of the ionospheric electric potential and ionospheric current vectors, respectively, for the five substorm epochs or phases. For these average patterns, each of the KRM output plots has been normalized to the specific values: quiet time, $-20 \mathrm{nT}$ in $\mathrm{AL}$ (71); growth phase, $-100 \mathrm{nT}$; expansion phase, $-250 \mathrm{nT}$; maximum epoch, $-500 \mathrm{nT}$; and recovery phase, $-250 \mathrm{nT}$ where AL (71) denotes the $\mathrm{AL}$ index value calculated from the $\mathrm{H}$ component value constructed from the data at 71 observatories.

In Fig. 2, the typical twin-vortex pattern in terms of the electrostatic potential can be identified, except for the quiet time. There are several points to be noted: (1) The dynamic range in the total potential difference is much smaller than that of the current density. For example, the ratio of the potential between quiet times and the peak substorm time is only $15 / 55$, which can be compared to $20 / 500$ in terms of the maximum electrojet current. This indicates that the electric field is relatively important during quiet times. (2) The difference in the total potential drop across the polar cap between the growth phase and the expansion phase is $2 \mathrm{kV}$ which is only a $7 \%$ increase, even though the difference in terms of the electrojet current is as large as $250 \%$. Similarly, the decrease in total potential from the peak to the recovery phase of substorms is only $9 \%$, when the corresponding current decreases considerably by $50 \%$ from 500 to 250 nT. These results clearly demonstrate that a large-scale electric field is dominant not only during the growth phase but also during the recovery phase of substorms. (3) The overall pattern becomes most complicated during the recovery phase of substorms, as the relative size of the high/low potential vortices varies with respect to the substorm phases. The negative potential area on the evening side is larger than the positive potential area on the morning side during the growth phase, but the situation is reversed at the peak epoch. The location of the peak potential is different at different substorm phases as well. With an increase in the electrojet current from the growth phase to the expansion phase, the peak of the

\section{ELECTRIC POTENTIAL}
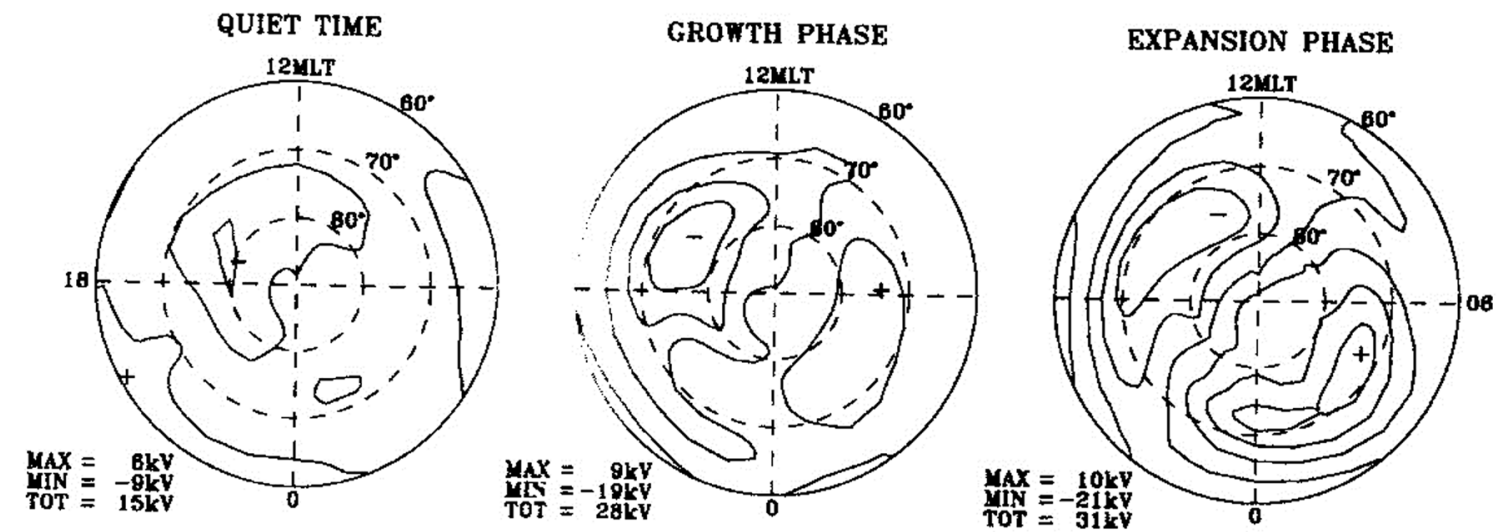

PEAK OF SLBSTORM
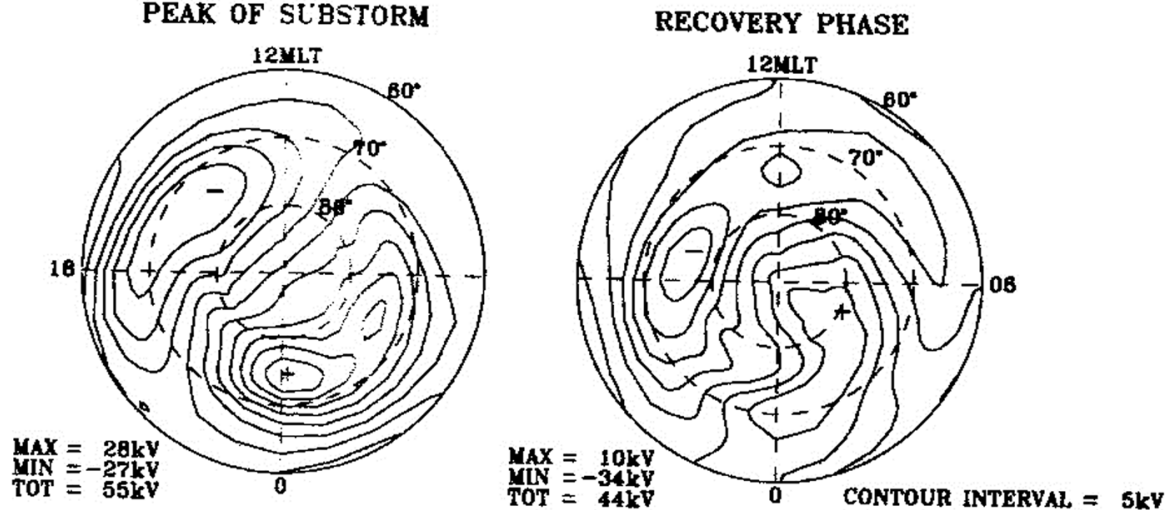

Fig. 2 Average distribution of the ionospheric electric potential for the five phases of substorms. The maximum and minimum potential values, as well as the total potential difference are shown for each diagram. Contour interval: 5 kV. Adapted from Kamide et al. (1996) 


\section{IONOSPHERIC CURRENT}
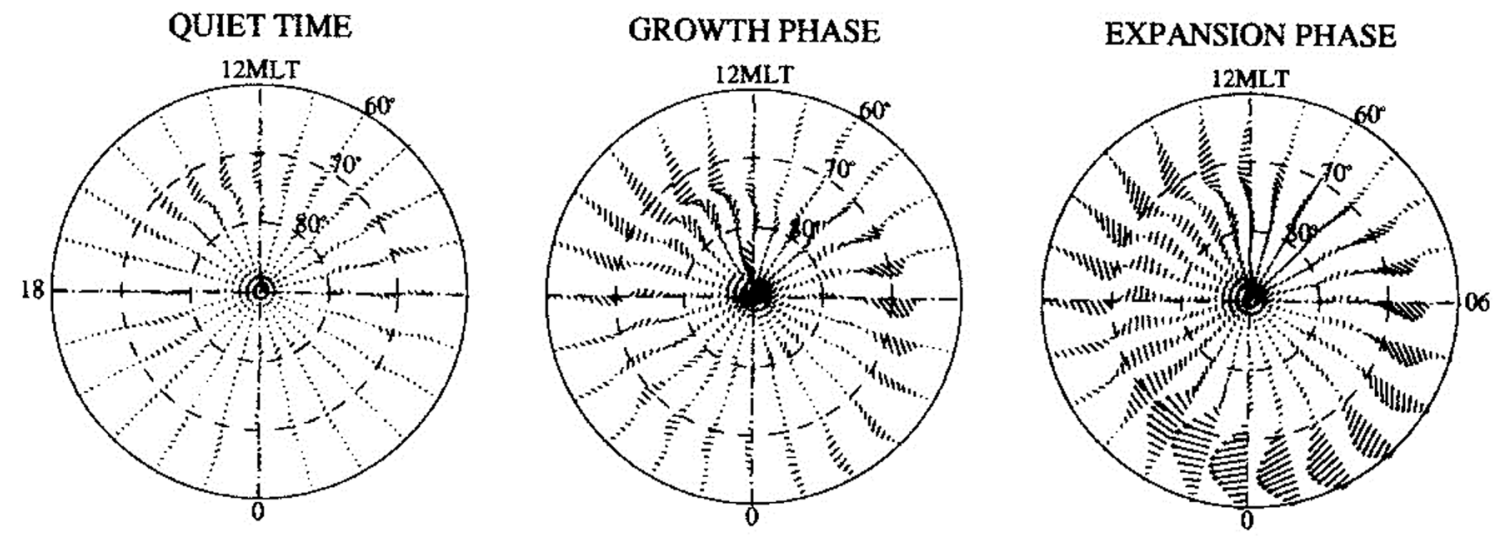

PEAK OF SUBSTORMS

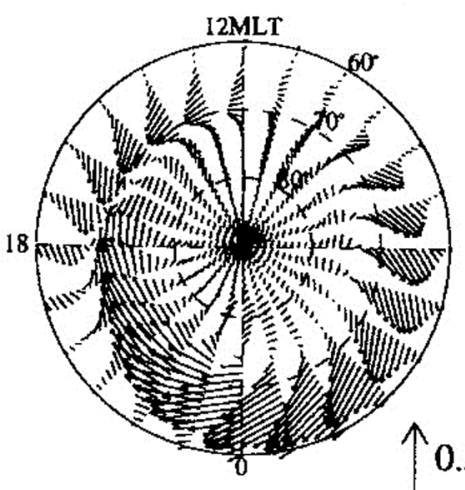

$0.5 \mathrm{Amp} / \mathrm{m}$

RECOVERY PHASE

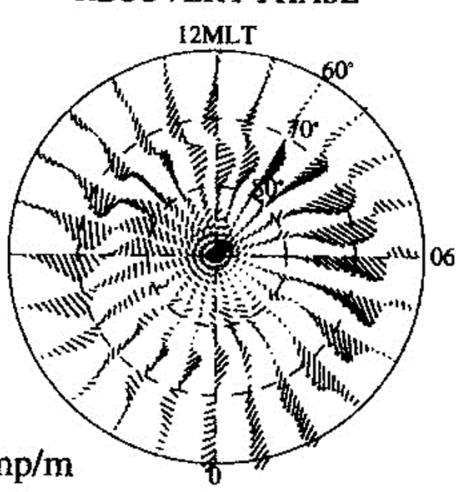

Fig. 3 Average distribution of the ionospheric current vectors for the five phases of substorms. The scale length of vectors is shown at the bottom of each diagram. Adapted from Kamide et al. (1996)

high potential vortex approaches the midnight sector, while the peak location of the low potential vortex seems to remain at approximately $15-16$ MLT.

Figure 3 shows the distribution of ionospheric current vectors corresponding to the five substorm phases shown in Fig. 2. During the growth phase of substorms, the eastward electrojet expands both latitudinally and longitudinally, and its intensity is almost doubled. The westward electrojet begins to grow during the growth phase and is centered at 0600 MLT. During the growth phase, the intensities of the eastward and westward electrojets are nearly equal. At the expansion onset of a substorm, however, the midnight portion of the westward electrojet is intensified dramatically from less than $0.1 \mathrm{~A} / \mathrm{m}$ before the expansion onset to nearly $0.4 \mathrm{~A} / \mathrm{m}$ after the onset; however, its morning portion remains almost the same. At the maximum epoch of substorms, the westward electrojet extends over a wide MLT range including the evening sector where it intrudes into the region poleward from the eastward electrojet.
We can recognize that the substorm westward electrojet peaks in the midnight sector, reaching $0.6 \mathrm{~A} / \mathrm{m}$, where the maximum $\mathrm{H}$ perturbation on the ground is $500 \mathrm{nT}$. It is significant that most of the changes in the current system occur between 1800 and 0600 MLT, like an addition of an intense westward current in the dark sector. During the recovery phase, the ionospheric current pattern becomes very much like that during the growth phase. The overall current system now consists of a pair of electrojets centered at dusk and dawn. However, one significant difference between the growthphase and recovery-phase patterns is that during the recovery phase, both electrojets are connected to the cusp currents on the dayside. That is, the eastward electrojet is contiguous to the dayside eastward current located on the equatorward side of the westward current, which then joins the morning side westward electrojet.

An important point to be confirmed is that there are essentially two modes to the westward electrojet, one in 
which the contributions to the electrojet magnitude are "conductivity dominant," and one in which they are "electric field dominant." The exact classification into these two modes during substorms using observed data is rather difficult, however, because the corresponding currents are contiguous everywhere in the electrojet. It has been shown statistically that the westward electrojet near midnight and in the early morning hours is characterized mainly by the relatively high Hall conductivity, whereas the westward electrojet in the late morning sector is dominated by the large southward electric field. The latter behavior is similar to that of the eastward electrojet in the evening sector, although the sense of the electric field is of course reversed.

To illustrate these points, a schematic diagram is presented in Fig. 4. The so-called Harang discontinuity is clearly manifested as a switch from the eastward electrojet to the westward electrojet, and more physically as a switch from the "electric field-rich" electrojet to the "conductivity-rich" electrojet. On the other hand, the boundary between the conductivity-dominant and the electric field-dominant westward electrojet in the morning hours is not as clear as indicated in Fig. 4 because the dominant driving mechanism changes across the boundary by keeping the current direction unchanged in this local time sector. Note that the locations of these boundaries are governed by the substorm phases as well.

Although it has been a common practice to assume that the westward electrojet during substorms is associated with a conductivity enhancement, a part of the westward electrojet in the later morning sector can in fact be intensified without having high conductivity values. This indicates that the electric field and the conductivity

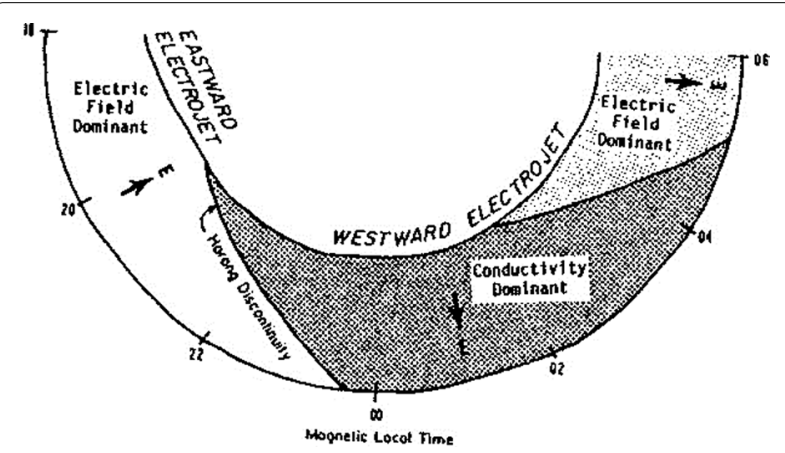

Fig. 4 Schematic illustration of the auroral electrojets, along with dominant direction of the electric fields. The Harang discontinuity separates the eastward electrojet to the west, and the westward electrojet to the east. This illustration indicates that there are two components within the westward electrojet: One is conductivity dominant in the midnight to early morning sector and the other is electric field dominant primarily in the late morning sector. Adapted from Kamide and Kokubun (1966) within the auroral electrojet are not in constant proportion in all locations. These characteristics of the relative function of the electric field and the conductance are extremely important because many researchers have tacitly assumed that the auroral electrojet, as the terminology implies, flows in the region of high auroral activity where the ionospheric conductivity is highest.

We contend that the possibility of there being two types of high-latitude current systems might have been implicit in some of the earlier studies that relied only on ground-based magnetic observations (Pytte et al. 1978). Two types of polar current systems, DP 1 and DP 2, have been proposed (Nishida and Kamide 1983). DP 2 consists of the eastward electrojet centered in the evening sector and the westward electrojet centered in the late morning sector, while DP 1 is dominated by the westward electrojet in the midnight sector. It is important to note that these two modes do coexist at all times during disturbed periods and that the relative strength of these two current systems varies from time to time, making individual current patterns very complicated.

These two components can be identified as the signatures for the directly driven and the unloading processes, respectively. The auroral electrojets associated with direct energy input from the solar wind into the magnetosphere flow primarily in the dusk and dawn sectors and increase in association with enhanced convection. The effect of this energy input has been described in terms of an enhancement of the DP 2 system. This is a typical signature for the substorm growth phase which precedes the expansion phase onset.

Unloading of energy stored in the tail during the growth phase leads to the formation of the intense westward electrojet in the midnight sector during the expansion phase of a substorm. This westward electrojet is controlled by high conductivity in the midnight sector, rather than a strong electric field. The enhancement in the conductivities, particularly the Hall conductivity, results from an enhanced ionization caused by accelerated electrons, reflecting the unloading of energy from the magnetotail. It is important to emphasize that the directly driven component is present throughout the entire lifetime of a substorm, indicating that the solar wind energy is continuously fed to the magnetosphere, though with varying degrees. This component becomes, therefore, relatively dominant again during the late recovery phase as the unloading component wanes.

\section{Discussion}

Historically, magnetic records obtained from the ground have widely been used to study physical processes that occur in the near-Earth environment. Over the last two hundred years, this type of study about the structure and 
temporal changes of the Sun-Earth space is now called space weather since the magnetic data obtained at the Earth's surface include much valuable information concerning various source currents. In fact, the study of space weather began as examining geomagnetic disturbances during geomagnetic storms. Other instruments, such as balloons, rockets, satellites, and radars, are all big in size, expensive to construct or install compared to inexpensive magnetometers. For this reason, scientists think that it is extremely difficult to separately evaluate the relative strength and importance of these currents in generating the particular modes of global or local magnetic disturbances that are to be studied in detail.

It is not too much to say that Kamide and coworkers have brought more than the KRM algorithm as a method and its applications to space research. They have studied the growth and decay of the ring current during magnetic storms. They have also examined the influence of the interplanetary magnetic field (IMF) on the ring current and substorm processes, finding that the IMF polarity determines not only the intensity but also the occurrence probability of substorms. They have used electron precipitation data from polar-orbiting satellites to identify the auroral belt. One of their findings from this extensive study has become a standard model of the size of the belt, which is controlled by the IMF and substorms.

Using the KRM method, it has been demonstrated that the auroral electrojet consists of two major components, i.e., the electric field-dominant and the conductivitydominant currents, and that their relative importance varies with substorm phases. It has been shown how the current and potential grow/decay in the ionosphere in response to changes in the solar wind. This method has proved to be quite useful for unifying the information obtained from synoptic observations of geomagnetic field with the comprehensive in situ observations by spacecraft. The usefulness of this method has been testified by numerous case studies. The KRM (and AMIE) method is now playing a key role even in real-time calculations in space weather predictions as well. This specification gives boundary conditions to other simulation schemes about the state of the magnetosphere, ionosphere, and thermosphere.

In a sense, the invention of magnetogram-inversion techniques can be treated as a revolution regarding the role of ground magnetic observations. These techniques help deriving the three-dimensional current system and global ionospheric potential pattern from ground-based magnetic observations. Using this inversion method, Kamide and coworkers have demonstrated that the auroral electrojet consists of two major components, i.e., the electric field-dominant and the conductivity-dominant currents, and that the balance of these components varies with substorm phases. They have also shown how the current and potential develop in the magnetosphere and ionosphere in response to variations in the solar wind. In other words, the development of the KRM method "elevated" geomagnetism to a quantitative measure of the state of the magnetosphere-ionosphere system.

Finally, we caution that the use of ground magnetic data to obtain successively magnetospheric and ionospheric parameters must go through a number of assumptions. The most serious assumption in the KRM algorithm is that one has to assume the distribution of the ionospheric conductance, but there are, at present, no measurements of the global ionospheric conductivities available in real time. Therefore, Kamide and colleagues utilized various models of the ionospheric conductance obtained, for example, by using incoherent scatter radars and satellite measurements of X-ray and UV auroras. We note that the obtained ionospheric currents are not very sensitive to the choice of the ionospheric conductances. Furthermore, in practically solving numerically the complicated equations of the KRM method, it is assumed that magnetic field lines are all vertical to the Earth's surface, simplifying the numerical scheme in calculating the electric potential. Limitations of the inversion techniques are described in more detail in Kamide et al. (1981).

\section{Authors' contributions}

YK prepared the first draft of the review paper. YK and NB together discussed and prepared the final version of the paper.

\section{Author details}

${ }^{1}$ Nagoya University, Nagoya 464-8601, Japan. ${ }^{2}$ Rikubetsu Space and Earth Science Museum, Rikubetsu 049-4301, Japan. ${ }^{3}$ Institute for Space and Earth Environment, Nagoya University, Nagoya 464-8601, Japan.

\section{Acknowledgements}

We thank the members of the 2015 AOGS Program Committee for encouraging us to write a concise review on this topic.

Competing interests

The authors have no competing interest in the publication of this article.

Received: 21 December 2015 Accepted: 15 February 2016

Published online: 10 March 2016

\section{References}

Ahn B-H, Kroehl HW, Kamide Y, Gorney DJ (1989) Estimation of ionospheric electrodynamic parameters using ionospheric conductance deduced from Bremsstrahlung X ray image data. J Geophys Res 94:2565-2586

Ahn B-H, Kamide Y, Kroehl HW, Gorney DJ (1992) Cross-polar cap potential difference, auroral electrojet indices, and solar wind parameters. J Geophys Res 97:1345-1352

Baumjohann W, Pellinen RJ, Opgenoorth HJ, Nielsen E (1981) Joint two-dimensional observations of ground magnetic and ionospheric electric field associated with auroral zone currents. 3. Current systems associated with local auroral break-ups. Planet Space Sci 29(431):1981

Fayermark DS (1977) Reconstruction of the three-dimensional current system of the high-latitude region from ground associated measurements. Geomag Aeron 17:114 
Feldstein Yl, Levitin AE (1986) Solar wind control of electric fields and currents in the ionosphere. J Geomag Geoelectr 38:1143

Glassmeier KH (1987) Ground-based observations of field-aligned currents in the auroral zone: methods and results. Ann Geophys 5:115-125

Kamide Y (1988) Estimate of electromagnetic quantities in space from ground magnetic records. Science 241:328-330

Kamide Y, Baumjohann W (1993) Magnetosphere-ionosphere coupling. Springer

Kamide Y, Kokubun S (1996) Two-component auroral electrojet: importance for substorm studies. J Geophys Res 101:13027-13046

Kamide Y, Richmond AD (1991) Ground-based studies of sensing magnetosphere/ionosphere interactions: convection and substorms. In: Proceedings of cluster Workshop, ESA SP-330, 1991

Kamide Y, Richmond AD, Matsushita S (1981) Estimation of ionospheric electric field, ionospheric currents and field-aligned currents from ground magnetic records. J Geophys Res 86:801

Kamide Y, Ishihara Y, Killeen TL, Craven JD, Frank LA, Heelis RA (1989) Combining electric field and aurora observations from DE 1 and 2 with ground magnetometer records to estimate ionospheric electromagnetic quantities. J Geophys Res 94:6723-6738

Kamide Y, Sun W, Akasofu S-I (1996) The average ionospheric electrodynamics for the different substorm phases. J Geophys Res 101:99-109
Kisabeth JL (1979) On calculating magnetic and vector potential fields due to large-scale magnetospheric current system and induced currents in an infinitely conducting earth. In: Olsen WP Quantitative modeling of magnetospheric processes. American Geophysical Union, pp 473

Mayaud PN (1980) Derivation, meaning, and use of geomagnetic indices. American Geophysical Union, Washington, DC

Mishin VM (1990) The magnetogram inversion technique and some applications. Space Sci Rev 53:83-163

Nishida A, Kamide Y (1983) Magnetospheric processes preceding the onset of an isolated substorm: a case study of the March 31, 1978, substorm. J Geophys Res 88:7005-7014

Pytte T, McPherron RL, Hones EW, West HL Jr (1978) Multiple satellite studies of magnetospheric substorms: distinction between polar magnetic substorms and convection-driven bays. J Geophys Res 83:663

Richmond AD, Kamide Y (1988) Mapping electrodynamic features of the highlatitude ionosphere from localized observations: technique. J Geophys Res 93:5741

Sugiura M (1964) Hourly values of equatorial Dst for the IGY. Ann Int Geophys Year 35:49

\section{Submit your manuscript to a SpringerOpen ${ }^{\odot}$ journal and benefit from:}

- Convenient online submission

- Rigorous peer review

- Immediate publication on acceptance

- Open access: articles freely available online

- High visibility within the field

- Retaining the copyright to your article

Submit your next manuscript at $\boldsymbol{s p r i n g e r o p e n . c o m ~}$ 